\title{
26. PETROLOGY AND GEOCHEMISTRY OF ROCKS FROM THE WALVIS RIDGE: DEEP SEA DRILLING PROJECT LEG 74, SITES 525, 527, AND 528 1
}

\author{
Geoffrey Thompson and Susan E. Humphris, Chemistry Department, Woods Hole Oceanographic Institution, \\ Woods Hole, Massachusetts
}

\begin{abstract}
Deep Sea Drilling Project Leg 74 drilled basement on the Walvis Ridge at Sites 525, 527, and 528. These sites are located on the crest and flanks of the segment of the Ridge about 68 to 70 m.y. old in the central province of the Ridge. Each site has a number of distinct subaqueous flows separated by sediment layers. Although variation in geochemistry among units and sites is related in part to alteration or crystal fractionation, some is caused by small-scale compositional variation in the mantle source of the basalts.

Leg 74 basalts are similar to other basalts recovered from the Walvis Ridge and the Rio Grande Rise. They show distinct compositional differences to mid-ocean ridge basalts in general, to those recovered from the South Atlantic at this latitude, and to basalts presently erupting in Tristan da Cunha. The composition of the Walvis Ridge basalts does not suggest simple mixtures of present-day MORB and Tristan da Cunha melts. If the Walvis Ridge represents the trace of the Tristan da Cunha hot spot as the plates separated, then the composition of the mantle source has differed at different times in the past, which suggests mantle heterogeneity.
\end{abstract}

\section{INTRODUCTION}

The Walvis Ridge is a linear discontinuous feature extending from the Mid-Atlantic Ridge, at about $38^{\circ} \mathrm{S}$, to the coastline of Africa, which it intersects near Cape Frio at $18^{\circ} \mathrm{S}$. It has been suggested that the Walvis Ridge represents a chain of volcanoes formed by plate movement over a fixed hot spot in the mantle (Wilson, 1965; Morgan, 1971, 1972; Detrick and Watts, 1979). Drilling evidence strongly suggests that the Walvis Ridge formed contemporaneously with the adjacent oceanic crust (Detrick et al., 1977).

Morphologically the Walvis Ridge can be divided into two distinct provinces-the Ridge and the Guyot provinces (Connary, 1972). The asymmetric Ridge province consists of a long, more or less continuous ridge section which can be subdivided into an eastern section, which strikes WSW from Cape Frio, and a central section, consisting of a system of en echelon, N-S-trending ridge segments progressively offset to the SW, as far as about $2^{\circ} \mathrm{E}$. The Guyot province lies between the main Walvis Ridge and Tristan da Cunha and is characterized by numerous isolated seamounts. The island of Tristan da Cunha is thought to be the modern-day representation of the mantle hot spot.

Leg 74 drilled three holes into basement on the Walvis Ridge at Sites 525, 527 and 528, all located in the central section of the Ridge. In this chapter we will describe the petrological and geochemical characteristics of basalts from each site, compare the sites, and compare the basalts with others from the Walvis Ridge, the Mid-Atlantic Ridge at this latitude, the Rio Grande Rise, and Tristan da Cunha. The implications for the origin and evolution of the Walvis Ridge will be the primary focus of this discussion.

${ }^{1}$ Moore, T. C., Jr., Rabinowitz, P. D., et al., Init. Repts. DSDP, 74: Washington (U.S. Govt. Printing Office).

\section{PREVIOUS WORK}

Basalts dredged from the eastern end of the Walvis Ridge have been reported (Hekinian, 1972; Hekinian and Thompson, 1976). These basalts are highly weathered but have chemical characteristics that show them to be tholeiitic in character, although much more enriched in incompatible elements such as $\mathrm{Ti}, \mathrm{K}, \mathrm{Sr}, \mathrm{Ba}$, and $\mathrm{Y}$ than mid-ocean ridge basalts. They are not as enriched or as alkalic as basalts from Tristan da Cunha. Judging by magnetic anomalies, these basalts came from crust about 110 m.y. old. Fodor, Keil, et al. (1977) reported on a trachytic tuff recovered from Site 359, Leg 39. This trachyte was about $40 \mathrm{~m}$.y. old and very similar to trachytes recovered from Tristan da Cunha, which suggests that at least part of the Walvis Ridge was compatible with a hot spot origin, although volcanism may have continued into the Eocene.

Humphris and Thompson (1982) have reported on a series of basalts dredged at many different locations along the Walvis Ridge. These basalts have tholeiitic and alkaline characteristics but are distinct from mid-ocean ridge basalts and from those of Tristan da Cunha. They noted that as a group the basalts from the eastern end of the Walvis Ridge had similar chemical characteristics, in particular a $\mathrm{Zr} / \mathrm{Nb}$ ratio of about 10 . Basalts from the central section had $\mathrm{Zr} / \mathrm{Nb}$ ratios close to 6 . $\mathrm{Zr} / \mathrm{Nb}$ ratios of basalts from the Guyot province ranged from about 8 to 4 ; basalts from Tristan da Cunha have $\mathrm{Zr} / \mathrm{Nb}$ ratios of about 3.5 . $\mathrm{Zr} / \mathrm{Nb}$ ratios are not greatly affected by fractional crystallization processes in magmas or changed by seawater alteration and are thus thought to be indicative of the mantle source region of the basalts (Erlank and Kable, 1976). These data suggest that the composition of the mantle source was different for the various provinces along the Walvis Ridge and may have changed progressively with time if the Ridge represents the trace of the Tristan da Cunha hot spot. 
Leg 72 drilling was done on the Rio Grande Rise, which is thought to be the western counterpart of the Walvis Ridge. Thompson et al. (in press) reported on basement basalts drilled at Hole $516 \mathrm{~F}$ and on dredged basalts previously discussed by Fodor, Hasler, et al. (1977). The Rio Grande Rise basalts from Hole 516F (83 m.y. old) are generally similar to basalts from the eastern end of the Walvis Ridge (80-100 m.y. old). The dredged basalts from the Rio Grande Rise are alkalic and similar to basalts and trachybasalts from Tristan da Cunha. Drilling during Leg 39 at Site 357 on the Rio Grande Rise recovered alkalic basalt breccias of middle Eocene age. Thompson et al. (in press) suggest that the Rio Grande Rise, like the Walvis Ridge, originated by seafloor spreading at or adjacent to a hot spot and that the hot spot influence continued through time, producing a linear series of volcanoes. They also suggest that the nature of the hot spot, or its influence on the spreading center, may have changed through time.

\section{DSDP LEG 74-SAMPLE LOCATIONS AND ANALYTICAL TECHNIQUE}

- The locations of Sites 525, 527, and 528 are shown in Figure 1, with other previously drilled or dredged locations. Samples from each site reported in this chapter are shown in Table 1. We have followed the volcanic unit numbering system of the shipboard log. Each unit represents a discrete series of basalt recoveries separated by sediment layers.

The samples were analyzed for major and trace elements by X-ray fluorescence spectroscopy following the technique of Schroeder et al. (1980). Precision and accuracy are of the order $\pm 1-3 \%$ for the major elements and about $\pm 5 \%$ for the trace elements. Ferrous iron was determined by titration following Jen (1973); $\mathrm{H}_{2} \mathrm{O}$ and $\mathrm{CO}_{2}$ were determined on a Perkin Elmer 240B CHN analyzer (Skinner et al., 1981). The analytical results are reported in Tables 2 through 7.

\section{DISCUSSION}

\section{Site 525}

Site 525 is located on the crest of a NNW-trending block of the Walvis Ridge. The site is on the edge of Magnetic Anomaly 32, which gives an age of about 70 m.y., in good agreement with a paleontologic age deduced from sediments just above basement and intercalated within discrete basalt flows of uppermost Campanian to lower Maestrichtian. This age is concordant with the adjacent basement and thus with an origin at or close to a spreading center. We penetrated $103 \mathrm{~m}$ of basement which, on the basis of sedimentary units di-

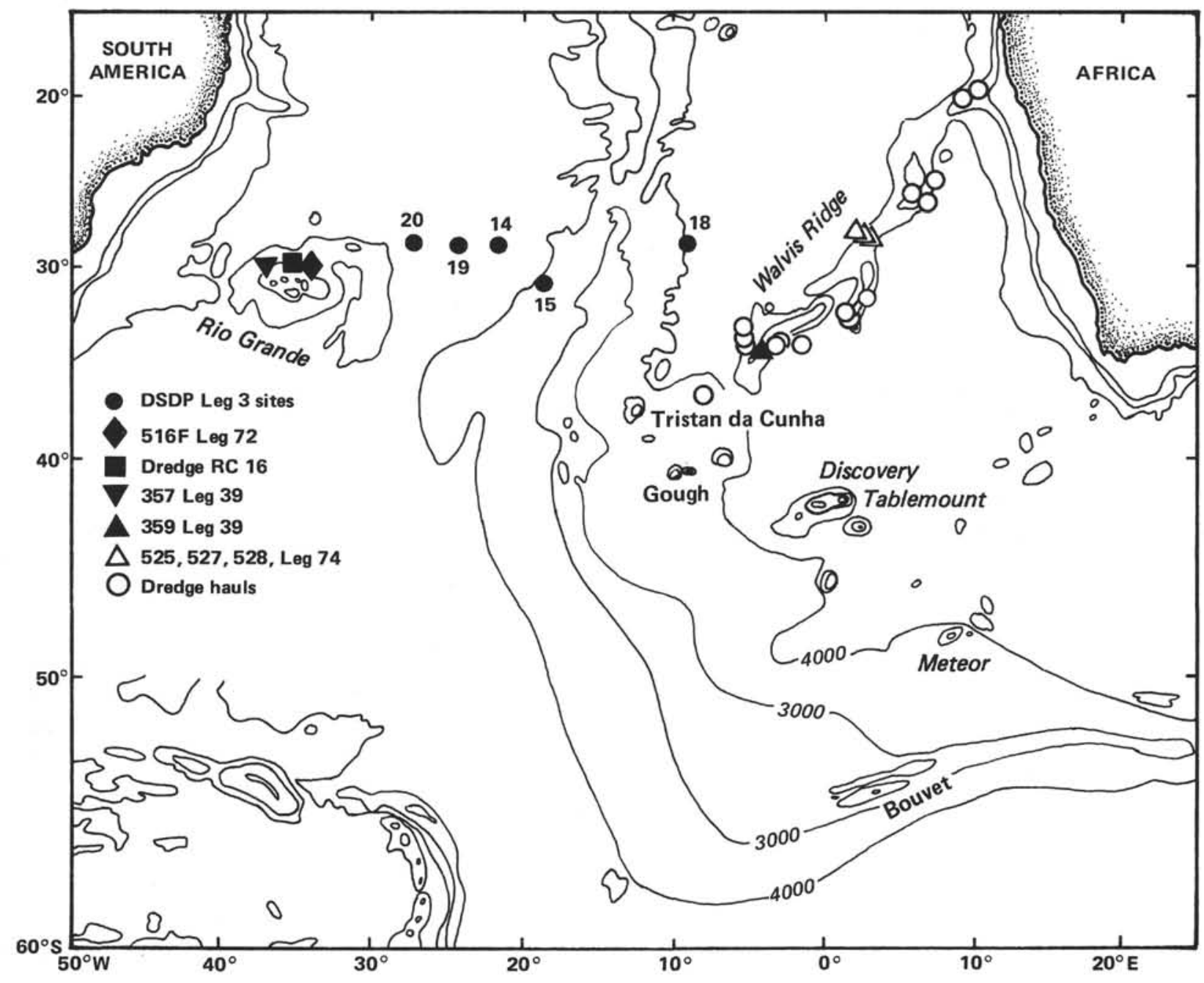

Figure 1. Map of the South Atlantic Region showing location of DSDP Sites 525, 527, and 528, Leg 74, and other DSDP sites and dredge hauls on the Walvis Ridge and Rio Grande Rise. 
Table 1. Sample locations, DSDP Sites 525, 527, and 528, Leg 74.

\begin{tabular}{|c|c|c|}
\hline Volcanic Unit & $\begin{array}{l}\text { Core/Section } \\
\text { (interval in cm) }\end{array}$ & Description \\
\hline \multicolumn{3}{|l|}{ Hole 525} \\
\hline \multirow[t]{3}{*}{1} & $53-2,96-101$ & $\begin{array}{l}\text { Highly altered, plagioclase with minor clinopy- } \\
\text { roxene }\end{array}$ \\
\hline & $53-3,107-112$ & $\begin{array}{l}\text { Highly altered, plagioclase with minor clinopy- } \\
\text { roxene }\end{array}$ \\
\hline & $54-2,50-56$ & $\begin{array}{l}\text { Highly altered, plagioclase with minor clinopy- } \\
\text { roxene }\end{array}$ \\
\hline 2 & $55-2,35-40$ & $\begin{array}{l}\text { Highly altered, plagioclase with minor clinopy- } \\
\text { roxene }\end{array}$ \\
\hline \multirow[t]{3}{*}{3} & $56-1,63-67$ & Vesicular, plagioclase with minor clinopyroxene \\
\hline & $56-4,5-11$ & Subophitic, plagioclase-pyroxene \\
\hline & $57-4,136-140$ & Subophitic, plagioclase-pyroxene \\
\hline \multirow{3}{*}{4} & $58-4,68-72$ & Pillow basalt, plagioclase-rich \\
\hline & $59-6,37-41$ & Pillow basalt, plagioclase-rich, very fine grained \\
\hline & $60-5,14-17$ & Pillow basalt, plagioclase-rich, very fine grained \\
\hline \multirow[t]{2}{*}{5} & $61-2,113-118$ & Vesicular plagioclase with minor clinopyroxene \\
\hline & $63-2,55-59$ & Vesicular plagioclase with minor clinopyroxene \\
\hline \multicolumn{3}{|l|}{ Hole 527} \\
\hline 1 & $39-2,92-96$ & $\begin{array}{l}\text { Phyric, plagioclase with minor pyroxene and } \\
\text { olivine }\end{array}$ \\
\hline 2 & $40-1,72-76$ & $\begin{array}{l}\text { Phyric, plagioclase with minor pyroxene and } \\
\text { olivine }\end{array}$ \\
\hline \multirow[t]{2}{*}{3} & $41-1,27-31$ & Plagioclase with minor pyroxene \\
\hline & $41-3,79-84$ & Plagioclase with minor pyroxene \\
\hline \multirow[t]{2}{*}{5} & $42-3,91-96$ & Plagioclase-clinopyroxene, fine grained \\
\hline & $44-4,62-67$ & Plagioclase-clinopyroxene, coarse grained \\
\hline \multicolumn{3}{|l|}{ Hole 528} \\
\hline \multirow[t]{2}{*}{1} & $39-1,122-126$ & Plagioclase-rich phyric, minor pyroxene \\
\hline & $40-5,69-74$ & Plagioclase-rich phyric, minor pyroxene \\
\hline 2 & $41-2,62-67$ & Fine-grained plagioclase-pyroxene \\
\hline 3 & $42-3,95-100$ & Similar to Unit I \\
\hline \multirow[t]{2}{*}{4} & $43-2,5-9$ & Similar to Unit I \\
\hline & $43-2,103-108$ & Similar to Unit I \\
\hline 5 & $44-2,64-68$ & Similar to Unit I \\
\hline 6 & $45-2,34-37$ & Similar to Unit I \\
\hline 7 & $47-1,56-61$ & Vesicular, plagioclase-pyroxene, altered \\
\hline 8 & $47-3,0-3$ & Vesicular, plagioclase-pyroxene, altered \\
\hline
\end{tabular}

Note: The volcanic units are as numbered in the shipboard descriptions.

viding discrete pillow basalt and massive flows, were divided into five volcanic units (see Table 1). The presence of pillow basalts with glassy margins indicates a subaqueous origin. The upper units 1 and 2 are more al- tered than the remaining units. In all the basalts plagioclase tends to dominate over augite.

\section{Site $\mathbf{5 2 7}$}

This site is located on the same block as Site 525 , but deeper on the northwestern flank. It is on Magnetic Anomaly 31 , and a middle Maestrichtian sediment layer lies just above basement, indicating an age of about 68 m.y. Basement is divided into 5 units, again on the basis of intercalated sediments, although in this case only 43 $\mathrm{m}$ of basement were penetrated. Units 1 and 2 contain plagioclase with minor olivine and pyroxene. Unit 3 is highly plagioclase phyric with sparse pyroxene and possibly some altered olivine phenocrysts. The basal Unit 5 , which makes up half the recovered sequence, is phyric and varies from fine- to coarse-grained with subophitic textures.

\section{Site 528}

This site is about halfway down the northwest flank of the same block as Site 525, midway between Sites 525 and 527 . The $80-\mathrm{m}$ basement complex is composed of at least eight different units divided by sediment layers. Age of basement is about $68 \mathrm{~m} . y$., judging by Magnetic Anomaly (31-32) and sediment age (middle Maestrichtian). Most of the units are medium- to coarse-grained highly plagioclase phyric basalts; Units 2,7 , and 8 are fine-grained plagioclase-pyroxene basalts with well-developed vesicles.

\section{Geochemical Variations within Individual Sites}

At Site 525, Volcanic Units 1 and 2 are similar but quite distinct from the remaining units in their chemical composition. Characteristically, Units 1 and 2 have higher concentrations of $\mathrm{Si}, \mathrm{Al}, \mathrm{Na}, \mathrm{V}, \mathrm{Cr}, \mathrm{Co}, \mathrm{Ni}, \mathrm{Cu}$, and $\mathrm{Zn}$ compared to Units 3, 4, and 5 and have lower concentrations of $\mathrm{Fe}, \mathrm{Mg}, \mathrm{Mn}, \mathrm{P}, \mathrm{Zr}$, and $\mathrm{Nb}$. Some of these differences may result from the higher degree of

Table 2. Major element analyses (wt. \%) of basalts for DSDP Hole 525A, Leg 74 (on volatile-free basis after ignition at $1000^{\circ} \mathrm{C}$ ).

\begin{tabular}{|c|c|c|c|c|c|c|c|c|c|c|c|c|c|}
\hline \multicolumn{14}{|l|}{ Volcanic } \\
\hline Unit & 1 & 1 & 1 & 2 & 3 & 3 & 3 & 4 & 4 & 4 & 4 & 5 & 5 \\
\hline $\begin{array}{l}\text { Core/Section } \\
\text { (interval in cm) }\end{array}$ & $\begin{array}{l}53-2, \\
96-101\end{array}$ & $\begin{array}{c}53-3 \\
107-112\end{array}$ & $\begin{array}{l}54-2 \\
50-56\end{array}$ & $\begin{array}{l}55-2 \\
35-40\end{array}$ & $\begin{array}{l}56-1 \\
63-67\end{array}$ & $\begin{array}{l}56-4, \\
5-11\end{array}$ & $\begin{array}{c}57-4 \\
136-140\end{array}$ & $\begin{array}{l}58-4 \\
68-72\end{array}$ & $\begin{array}{c}58-4 \\
139-144\end{array}$ & $\begin{array}{l}59-6 \\
37-41\end{array}$ & $\begin{array}{l}60-5 \\
14-17\end{array}$ & $\begin{array}{l}\text { 61-2, } \\
113-118\end{array}$ & $\begin{array}{c}63-2, \\
55-59\end{array}$ \\
\hline \multicolumn{14}{|l|}{ Major elements } \\
\hline $\mathrm{SiO}_{2}$ & 54.64 & 51.34 & 55.34 & 53.91 & 50.43 & 51.21 & 50.78 & 50.57 & 51.37 & 52.32 & 52.17 & 52.10 & 51.06 \\
\hline $\mathrm{TiO}_{2}$ & 2.86 & 2.68 & 2.82 & 3.09 & 2.97 & 2.46 & 2.76 & 2.96 & 2.92 & 3.11 & 3.26 & 2.92 & 3.19 \\
\hline $\mathrm{Al}_{2} \overline{\mathrm{O}}_{3}$ & 21.89 & 21.30 & 21.37 & 22.66 & 16.39 & 14.71 & 16.23 & 16.56 & 15.91 & 16.68 & 18.22 & 15.54 & 15.54 \\
\hline $\mathrm{FeO}^{\mathrm{a}}$ & 4.51 & 6.14 & 4.36 & 4.55 & 9.56 & 11.43 & 10.83 & 11.74 & 10.89 & 9.42 & 10.72 & 12.67 & 11.61 \\
\hline $\mathrm{MnO}$ & 0.03 & 0.08 & 0.01 & 0.01 & 0.10 & 0.13 & 0.11 & 0.11 & 0.12 & 0.10 & 0.07 & 0.10 & 0.12 \\
\hline $\mathrm{MgO}$ & 0.89 & 1.10 & 1.13 & 1.51 & 4.47 & 5.18 & 4.52 & 4.38 & 5.15 & 3.99 & 3.05 & 4.63 & 5.42 \\
\hline $\mathrm{CaO}$ & 8.40 & 10.55 & 7.31 & 8.03 & 10.62 & 9.90 & 9.47 & 9.12 & 9.47 & 8.82 & 6.63 & 6.04 & 7.93 \\
\hline $\mathrm{Na}_{2} \mathrm{O}$ & 4.33 & 4.20 & 4.10 & 3.65 & 3.09 & 2.75 & 3.26 & 3.13 & 3.01 & 3.40 & 3.51 & 3.06 & 2.96 \\
\hline $\mathrm{K}_{2} \mathrm{O}$ & 1.18 & 0.97 & 1.54 & 0.53 & 0.48 & 1.01 & 1.15 & 0.64 & 0.56 & 0.92 & 0.78 & 1.59 & 0.88 \\
\hline $\mathrm{P}_{2} \mathrm{O}_{5}$ & 0.20 & 0.16 & 0.25 & 0.18 & 0.25 & 0.29 & 0.30 & 0.31 & 0.28 & 0.32 & 0.35 & 0.59 & 0.45 \\
\hline Sum & 98.93 & 98.52 & 98.23 & 98.12 & 98.36 & 99.07 & 99.41 & 99.52 & 99.68 & 99.08 & 98.76 & 99.24 & 99.16 \\
\hline \multicolumn{14}{|l|}{ Other analyses ${ }^{b}$} \\
\hline $\mathrm{FeO}$ & 1.38 & 2.87 & 1.05 & 0.49 & 3.79 & 6.13 & 5.04 & 5.43 & 4.93 & 4.14 & 3.65 & 4.24 & 5.12 \\
\hline $\mathrm{Fe}_{2} \mathrm{O}_{3}$ & 3.48 & 3.63 & 3.68 & 4.51 & 6.41 & 5.89 & 6.43 & 7.01 & 6.62 & 5.87 & 7.86 & 9.37 & 7.21 \\
\hline $\mathrm{H}_{2} \mathrm{O}^{+}$ & 1.01 & 1.13 & 1.28 & 2.22 & 1.57 & 1.12 & 1.15 & 1.59 & 1.54 & 1.33 & 2.12 & 1.98 & 1.50 \\
\hline $\mathrm{H}_{2} \mathrm{O}^{-}$ & 3.13 & 2.29 & 2.67 & 6.30 & 3.26 & 2.09 & 2.48 & 3.22 & 2.81 & 2.35 & 4.88 & 4.16 & 3.15 \\
\hline $\mathrm{CO}_{2}$ & 1.25 & 4.03 & 0.24 & 0.27 & 1.88 & 2.25 & 1.68 & 2.71 & 0.59 & 0.47 & 0.88 & 0.11 & 0.19 \\
\hline
\end{tabular}

a Total $\mathrm{Fe}$ as $\mathrm{FeO}$

b $\mathrm{On}$ a dried $\left(110^{\circ} \mathrm{C}\right)$ basis, except $\mathrm{H}_{2} \mathrm{O}^{-}$. 
Table 3. Trace element analyses (ppm) of basalts for DSDP Hole 525A, Leg 74 (on a dried, $110^{\circ} \mathrm{C}$ basis).

\begin{tabular}{|c|c|c|c|c|c|c|c|c|c|c|c|c|c|}
\hline $\begin{array}{c}\text { Volcanic } \\
\text { Unit }\end{array}$ & 1 & 1 & 1 & 2 & 3 & 3 & 3 & 4 & 4 & 4 & 4 & 5 & 5 \\
\hline $\begin{array}{l}\text { Core/Section } \\
\text { (interval in } \mathrm{cm} \text { ) }\end{array}$ & $\begin{array}{l}53-2 \\
96-101\end{array}$ & $\begin{array}{c}53-3 \\
107-112\end{array}$ & $\begin{array}{l}54-2 \\
50-56\end{array}$ & $\begin{array}{l}55-2 \\
35-40\end{array}$ & $\begin{array}{l}56-1 \\
63-67\end{array}$ & $\begin{array}{l}56-4 \\
5-11\end{array}$ & $\begin{array}{c}57-4 \\
136-140\end{array}$ & $\begin{array}{l}58-4 \\
68-72\end{array}$ & $\begin{array}{c}58-4 \\
139-144\end{array}$ & $\begin{array}{l}59-6 \\
37-41\end{array}$ & $\begin{array}{l}60-5 \\
14-17\end{array}$ & $\begin{array}{c}61-2 \\
113-118\end{array}$ & $\begin{array}{r}63-2, \\
55-59\end{array}$ \\
\hline$R b$ & 31 & 23 & 42 & 7.3 & 4.6 & 18.5 & 20 & 4.9 & 3.9 & 8.7 & 6.5 & 20 & 5.4 \\
\hline V & 516 & 480 & 466 & 457 & 399 & 303 & 394 & 410 & 397 & 403 & 432 & 355 & 366 \\
\hline $\mathrm{Cr}$ & 385 & 344 & 338 & 389 & 32 & 30 & 48 & 33 & 39 & 48 & 49 & 36 & 35 \\
\hline Co & 127 & 99 & 63 & 127 & 40 & 38 & 46 & 29 & 43 & 66 & 46 & 25 & 50 \\
\hline $\mathrm{Ni}$ & 62 & 73 & 43 & 153 & 35 & 33 & 38 & 39 & 42 & 59 & 46 & 27 & 42 \\
\hline $\mathrm{Cu}$ & 112 & 99 & 126 & 110 & 63 & 63 & 66 & 72 & 76 & 80 & 77 & 39 & 46 \\
\hline $\mathrm{Zn}$ & 248 & 128 & 436 & 37 & 117 & 80 & 94 & 115 & 100 & 135 & 94 & 123 & 102 \\
\hline $\mathrm{Sr}$ & 433 & 422 & 428 & 578 & 521 & 427 & 467 & 488 & 465 & 501 & 540 & 483 & 511 \\
\hline $\mathrm{Ba}$ & 375 & 388 & 365 & 295 & 452 & 370 & 433 & 386 & 378 & 411 & 411 & 472 & 503 \\
\hline $\mathrm{Y}$ & 32 & 35 & 36 & 28 & 36 & 33 & 34 & 38 & 39 & 52 & 37 & 43 & 43 \\
\hline $\mathrm{Zr}$ & 222 & 228 & 215 & 209 & 246 & 214 & 241 & 245 & 246 & 263 & 283 & 314 & 332 \\
\hline $\mathrm{Nb}$ & 17 & 17 & 17 & 19 & 25 & 22 & 25 & 24 & 24 & 24 & 28 & 30 & 31 \\
\hline
\end{tabular}

Table 4. Major element analyses (wt.\%) of basalts from DSDP Hole 527 , Leg 74 (on volatile-free basis after ignition at $1000^{\circ} \mathrm{C}$ ).

\begin{tabular}{|c|c|c|c|c|c|c|}
\hline $\begin{array}{c}\text { Volcanic } \\
\text { Unit }\end{array}$ & 1 & 2 & 3 & 3 & 5 & 5 \\
\hline $\begin{array}{l}\text { Core/Section } \\
\text { (interval in } \mathrm{cm} \text { ) }\end{array}$ & $\begin{array}{l}39-2 \\
92-96\end{array}$ & $\begin{array}{l}40-1 \\
72-76\end{array}$ & $\begin{array}{l}41-1 \\
27-31\end{array}$ & $\begin{array}{l}41-3 \\
79-84\end{array}$ & $\begin{array}{l}42-3 \\
91-96\end{array}$ & $\begin{array}{l}44-4, \\
62-67\end{array}$ \\
\hline \multicolumn{7}{|l|}{ Major elements } \\
\hline $\mathrm{SiO}_{2}$ & 50.78 & 50.05 & 49.96 & 49.80 & 50.22 & 49.80 \\
\hline $\mathrm{TiO}_{2}$ & 1.84 & 1.79 & 1.14 & 1.17 & 2.52 & 2.49 \\
\hline $\mathrm{Al}_{2} \mathrm{O}_{3}$ & 14.00 & 14.13 & 17.49 & 17.04 & 14.81 & 14.51 \\
\hline $\mathrm{FeO}^{\mathrm{a}}$ & 13.21 & 13.44 & 10.02 & 10.24 & 12.59 & 12.89 \\
\hline $\mathrm{MnO}$ & 0.17 & 0.22 & 0.18 & 0.18 & 0.21 & 0.20 \\
\hline $\mathrm{MgO}$ & 6.82 & 6.52 & 6.22 & 6.17 & 6.88 & 5.80 \\
\hline $\mathrm{CaO}$ & 9.69 & 10.81 & 12.81 & 12.73 & 6.96 & 9.31 \\
\hline $\mathrm{Na}_{2} \mathrm{O}$ & 2.54 & 2.34 & 2.23 & 2.98 & 2.98 & 2.96 \\
\hline $\mathrm{K}_{2} \mathrm{O}$ & 0.51 & 0.27 & 0.21 & 0.18 & 1.36 & 1.02 \\
\hline $\mathrm{P}_{2} \mathrm{O}_{5}$ & 0.22 & 0.20 & 0.10 & 0.12 & 0.46 & 0.38 \\
\hline Sum & 99.78 & 99.77 & 100.31 & 99.86 & 98.99 & 99.36 \\
\hline \multicolumn{7}{|l|}{ Other analyses $b$} \\
\hline $\mathrm{Fe}$ & 7.34 & 8.89 & 6.62 & 7.05 & 6.48 & 7.29 \\
\hline $\mathrm{Fe}_{2} \mathrm{O}_{3}$ & 6.52 & 5.06 & 3.78 & 3.55 & 6.79 & 6.22 \\
\hline $\mathrm{H}_{2} \mathrm{O}^{+}$ & 0.80 & 0.77 & 0.56 & 0.57 & 1.28 & 0.96 \\
\hline $\mathrm{H}_{2} \mathrm{O}^{-}$ & 1.91 & 1.18 & 0.57 & 0.57 & 2.67 & 2.24 \\
\hline $\mathrm{CO}_{2}$ & 0.07 & 0.07 & 0.09 & 0.10 & 0.12 & 0.08 \\
\hline
\end{tabular}

a Total $\mathrm{Fe}$ as $\mathrm{FeO}$.

b $\mathrm{On}$ a dried $\left(110^{\circ} \mathrm{C}\right)$ basis, except $\mathrm{H}_{2} \mathrm{O}^{-}$.

Table 5. Trace element analyses (ppm) of basalts from DSDP Hole 527 , Leg 74 (on a dried, $110^{\circ} \mathrm{C}$ basis).

\begin{tabular}{ccccccc}
\hline $\begin{array}{c}\text { Volcanic } \\
\text { Unit }\end{array}$ & 1 & 2 & 3 & 3 & 5 & \multicolumn{1}{c}{5} \\
\hline $\begin{array}{c}\text { Core/Section } \\
\text { (interval in cm) }\end{array}$ & $39-2$, & $40-1$, & $41-1$, & $41-3$, & $42-3$, & $44-4$, \\
\hline $\mathrm{Rb}$ & 10.5 & $32-76$ & $27-31$ & $79-84$ & $91-96$ & $62-67$ \\
$\mathrm{~V}$ & 425 & 422 & 27.5 & 3.9 & 26 & 19 \\
$\mathrm{Cr}$ & 45 & 61 & 70 & 64 & 378 & 366 \\
$\mathrm{Co}$ & 43 & 43 & 40 & 41 & 41 & 18 \\
$\mathrm{Ni}$ & 49 & 57 & 56 & 53 & 26 & 29 \\
$\mathrm{Cu}$ & 197 & 195 & 144 & 147 & 76 & 94 \\
$\mathrm{Zn}$ & 87 & 91 & 63 & 70 & 86 & 85 \\
$\mathrm{Sr}$ & 154 & 158 & 150 & 151 & 305 & 340 \\
$\mathrm{Ba}$ & 172 & 133 & 91 & 112 & 366 & 407 \\
$\mathrm{Y}$ & 42 & 42 & 30 & 31 & 43 & 44 \\
$\mathrm{Zr}$ & 118 & 116 & 76 & 77 & 185 & 178 \\
$\mathrm{Nb}$ & 14 & 13 & 7.6 & 7.2 & 30 & 29 \\
\hline
\end{tabular}

alteration of these two units. However, the observation that some of the incompatible element concentrations are low suggests Units 1 and 2 are not as highly evolved as the other units. The $\mathrm{Zr} / \mathrm{Nb}$ ratios are a little higher -11 to 13 in Units 1 and 2 compared to about 10 for the other units-which suggests distinct and different magma sources. Of the remaining units, Volcanic Unit 5 appears more evolved than Units 3 and 4, judging by the higher concentrations of incompatible elements such as $\mathrm{K}, \mathrm{Fe}$, $\mathrm{P}, \mathrm{Zr}, \mathrm{Nb}, \mathrm{Ba}$, and $\mathrm{Y}$.

At Site 527 Volcanic Units 1 and 2 are geochemically similar. Unit 3 is dominated by the presence of plagioclase seen in the high $\mathrm{Ca}$ and $\mathrm{Al}$ concentrations. It also appears less evolved than Units 1 and 2, based on the lower $\mathrm{FeO} / \mathrm{MgO}$ ratio (1.6 compared to 2 ) and lower concentrations of incompatible elements such as $\mathrm{Ti}, \mathrm{Fe}$, $\mathrm{K}, \mathrm{P}, \mathrm{V}, \mathrm{Ba}, \mathrm{Y}, \mathrm{Zr}$, and $\mathrm{Nb}$. The $\mathrm{Zr} / \mathrm{Nb}$ ratio is 10 to 11 compared to 8 to 9 for Units 1 and 2, which suggests slightly different magmatic sources. Unit 5 is quite distinct from the other units in its higher concentration of $\mathrm{Ti}, \mathrm{Na}, \mathrm{K}, \mathrm{P}, \mathrm{Sr}, \mathrm{Ba}, \mathrm{Zr}$, and $\mathrm{Nb}$. Although it has lower concentrations of compatible elements such as $\mathrm{Cr}, \mathrm{Ni}$, and $\mathrm{Cu}$, it also has lower concentrations of $\mathrm{Fe}$ and a $\mathrm{Zr} / \mathrm{Nb}$ ratio of only 6 , which suggests that these differences are not related to fractional crystallization alone.

At Site 528 Volcanic Units 2, 7, and 8 are quite distinct from the remainder, being much more highly evolved-i.e., having higher concentrations of incompatible elements such as $\mathrm{Ti}, \mathrm{Fe}, \mathrm{K}, \mathrm{P}, \mathrm{V}, \mathrm{Sr}, \mathrm{Ba}, \mathrm{Y}, \mathrm{Zr}$, and $\mathrm{Nb}$ and lower concentration of compatible elements such as $\mathrm{Mg}, \mathrm{Cr}, \mathrm{Ni}$, and $\mathrm{Cu}$. Of the remaining units, Unit 1 is probably the least evolved. All the units except for Unit 2, which has a $\mathrm{Zr} / \mathrm{Nb}$ ratio of about 5 , have $\mathrm{Zr} / \mathrm{Nb}$ ratios 6 to 7 , which suggests they are derived from a similar source.

\section{Geochemical Variations among Sites}

In general Site 525 at the crest of the Walvis Ridge is distinct from the other sites. Figure 2, a plot of total alkali versus $\mathrm{SiO}_{2}$, shows that Site 525 tends to be more Si-rich and, except for the more evolved or altered samples from other sites, to have a higher alkali content. Figure 3 plots the $\mathrm{TiO}_{2}$ contents of the different sites versus a number of incompatible elements. Clearly Site 525 is generally more titanium- and zirconium-rich than the other sites. Sites 527 and 528, except for the least 
Table 6. Major element analyses (wt.\%) of basalts from DSDP Hole 528, Leg 74 (on a volatile-free basis after ignition at $1000^{\circ} \mathrm{C}$ ).

\begin{tabular}{|c|c|c|c|c|c|c|c|c|c|c|}
\hline Volcanic & & & & & & & & & & \\
\hline Unit & 1 & 1 & 2 & 3 & 4 & 4 & 5 & 6 & 7 & 8 \\
\hline $\begin{array}{c}\text { Core/Section } \\
\text { (interval in cm) }\end{array}$ & $\begin{array}{c}39-1, \\
122-126\end{array}$ & $\begin{array}{l}40-5 \\
69-74\end{array}$ & $\begin{array}{l}41-2, \\
62-67\end{array}$ & $\begin{array}{l}42-3, \\
95-100\end{array}$ & $\begin{array}{c}43-2 \\
5-9\end{array}$ & $\begin{array}{c}43-2 \\
103-108\end{array}$ & $\begin{array}{l}44-2 \\
64-68\end{array}$ & $\begin{array}{l}45-2 \\
34-37\end{array}$ & $\begin{array}{l}47-1 \\
56-61\end{array}$ & $\begin{array}{c}47-3 \\
0-3\end{array}$ \\
\hline \multicolumn{11}{|l|}{ Major elements } \\
\hline $\mathrm{SiO}_{2}$ & 50.31 & 49.82 & 50.71 & 50.16 & 50.41 & 49.78 & 49.83 & 49.64 & 49.14 & 50.08 \\
\hline $\mathrm{TiO}_{2}$ & 1.32 & 1.22 & 2.81 & 1.35 & 1.59 & 1.43 & 1.90 & 1.91 & 2.85 & 2.89 \\
\hline $\mathrm{Al}_{2} \mathrm{O}_{3}$ & 17.09 & 17.16 & 16.11 & 18.08 & 17.43 & 17.41 & 15.23 & 16.52 & 14.75 & 14.81 \\
\hline $\mathrm{FeO}^{\mathrm{a}}$ & 9.04 & 9.60 & 11.89 & 9.73 & 9.71 & 10.17 & 11.98 & 11.34 & 13.31 & 13.46 \\
\hline $\mathrm{MnO}$ & 0.14 & 0.17 & 0.24 & 0.15 & 0.21 & 0.15 & 0.20 & 0.24 & 0.24 & 0.22 \\
\hline $\mathrm{MgO}$ & 7.17 & 6.75 & 5.78 & 6.31 & 6.15 & 6.09 & 6.40 & 6.25 & 5.80 & 5.90 \\
\hline $\mathrm{CaO}$ & 11.47 & 12.55 & 5.52 & 12.06 & 11.05 & 12.07 & 9.41 & 9.82 & 8.74 & 7.08 \\
\hline $\mathrm{Na}_{2} \mathrm{O}$ & 2.73 & 2.26 & 3.48 & 2.49 & 2.77 & 2.33 & 2.90 & 2.74 & 3.15 & 3.34 \\
\hline $\mathrm{K}_{2} \mathrm{O}$ & 0.63 & 0.41 & 2.45 & 0.36 & 0.73 & 0.50 & 1.05 & 1.16 & 1.39 & 1.42 \\
\hline $\mathrm{P}_{2} \mathrm{O}_{5}$ & 0.17 & 0.13 & 0.56 & 0.13 & 0.18 & 0.15 & 0.29 & 0.23 & 0.44 & 0.52 \\
\hline Sum & 100.07 & 100.07 & 99.55 & 100.82 & 100.23 & 100.08 & 99.19 & 99.85 & 99.81 & 99.72 \\
\hline \multicolumn{11}{|l|}{ Other analyses $b$} \\
\hline $\mathrm{FeO}$ & 4.93 & 5.61 & 5.22 & 4.92 & 4.87 & 5.53 & 5.61 & 6.01 & 6.02 & 5.79 \\
\hline $\mathrm{Fe}_{2} \mathrm{O}_{3}$ & 4.57 & 4.43 & 7.41 & 5.35 & 5.38 & 5.16 & 7.08 & 5.92 & 8.10 & 8.52 \\
\hline $\mathrm{H}_{2} \mathrm{O}^{+}$ & 0.74 & 1.15 & 1.27 & 0.97 & 1.24 & 0.94 & 1.31 & 1.67 & 0.97 & 1.03 \\
\hline $\mathrm{H}_{2} \mathrm{O}^{-}$ & 1.02 & 1.18 & 2.55 & 1.94 & 2.14 & 1.57 & 2.01 & 1.37 & 2.56 & 2.35 \\
\hline $\mathrm{CO}_{2}$ & 0.46 & 0.09 & 0.20 & 0.23 & 0.31 & 0.23 & 0.52 & 0.36 & 0.92 & 0.21 \\
\hline
\end{tabular}

Total $\mathrm{Fe}$ as $\mathrm{FeO}$.

b $\mathrm{On}$ a dried $\left(110^{\circ} \mathrm{C}\right)$ basis, except $\mathrm{H}_{2} \mathrm{O}^{-}$.

Table 7. Trace element analyses (ppm) of basalts from DSDP Hole 528, Leg 74 (on a dried, $110^{\circ} \mathrm{C}$ basis).

\begin{tabular}{|c|c|c|c|c|c|c|c|c|c|c|}
\hline $\begin{array}{l}\text { Volcanic } \\
\text { Unit }\end{array}$ & 1 & 1 & 2 & 3 & 4 & 4 & 5 & 6 & 7 & 8 \\
\hline $\begin{array}{l}\text { Core/Section } \\
\text { (interval in cm) }\end{array}$ & $\begin{array}{c}39-1 \\
122-126\end{array}$ & $\begin{array}{l}40-5 \\
69-74\end{array}$ & $\begin{array}{l}41-2 \\
62-67\end{array}$ & $\begin{array}{l}42-3 \\
95-100\end{array}$ & $\begin{array}{c}43-2 \\
5-9\end{array}$ & $\begin{array}{c}43-2 \\
103-108\end{array}$ & $\begin{array}{l}44-2 \\
64-68\end{array}$ & $\begin{array}{l}45-2 \\
34-37\end{array}$ & $\begin{array}{l}47-1 \\
56-61\end{array}$ & $\begin{array}{c}47-3 \\
0-3\end{array}$ \\
\hline $\mathrm{Rb}$ & 14 & 10 & 40 & 4.7 & 15 & 6.7 & 17 & 18 & 17 & 20 \\
\hline V & 312 & 258 & 441 & 353 & 340 & 309 & 353 & 368 & 466 & 457 \\
\hline $\mathrm{Cr}$ & 42 & 48 & 3 & 74 & 49 & 48 & 26 & 25 & 23 & 15 \\
\hline Co & 45 & 41 & 39 & 48 & 50 & 44 & 42 & 44 & 44 & 34 \\
\hline $\mathrm{Ni}$ & 53 & 52 & 18 & 59 & 55 & 50 & 43 & 41 & 26 & 24 \\
\hline $\mathrm{Cu}$ & 129 & 137 & 45 & 133 & 158 & 148 & 116 & 130 & 70 & 63 \\
\hline $\mathrm{Zn}$ & 48 & 54 & 100 & 74 & 77 & 73 & 78 & 78 & 104 & 101 \\
\hline $\mathrm{Sr}$ & 283 & 255 & 380 & 243 & 296 & 307 & 298 & 292 & 337 & 334 \\
\hline $\mathrm{Ba}$ & 201 & 188 & 667 & 196 & 256 & 231 & 334 & 323 & 526 & 579 \\
\hline$Y$ & 25 & 27 & 43 & 32 & 29 & 28 & 38 & 33 & 44 & 47 \\
\hline $\mathrm{Z}_{r}$ & 98 & 89 & 240 & 98 & 114 & 106 & 158 & 138 & 186 & 196 \\
\hline $\mathrm{Nb}$ & 13 & 12 & 51 & 14 & 19 & 17 & 26 & 22 & 31 & 33 \\
\hline
\end{tabular}

evolved units of Site 527, are generally similar to one another. Figure 4 plots $\mathrm{Zr}$ versus $\mathrm{Ba}$ and $\mathrm{Nb}$ where the differences between the sites are more distinctly seen. The elements $\mathrm{Zr}$ and $\mathrm{Nb}$ in particular are least affected by alteration. Site 525 is quite distinctive with respect to these two element abundances. In terms of $\mathrm{Zr} / \mathrm{Nb}$ ratio, Site 525 has ratios of 10 or greater. Only Volcanic Units 1,2 , and 3 of Site 527 have ratios close to 10 ; all the remaining units from Site 527 and all from Site 528 have ratios closer to 6 .

\section{Regional Geochemical Comparison}

Table 8 shows the results of previous basalt analyses from the Walvis Ridge, Rio Grande Rise, Tristan da Cunha, and the Mid-Atlantic Ridge at this latitude. Clearly Sites 525,527 , and 528 are distinct from presentday mid-ocean ridge basalts and from those from $30^{\circ} \mathrm{S}$, which range in age up to $50 \mathrm{~m} . \mathrm{y}$. and were drilled on Leg 3 (Frey et al., 1974). Basalts from the Walvis Ridge sites are apparently not as enriched in incompatible elements as basalts from Tristan da Cunha. However, they do have many similarities with other basalts reported from the Walvis Ridge and from the Rio Grande Rise. Figure
5 shows a plot of $\mathrm{Zr}$ versus $\mathrm{Nb}$ for many previously studied samples from this region of the South Atlantic as well as for those at Sites 525, 527, and 528. This figure suggests some interesting relationships. MORBs generally have high $\mathrm{Zr} / \mathrm{Nb}-20$ or greater - and appear to represent a distinct mantle source. Basalts from Tristan da Cunha, the present-day hot spot, have $\mathrm{Zr} / \mathrm{Nb}$ ratios close to 3.5 and are quite distinct. Basalts from the Walvis Ridge are somewhat variable between these two extreme sources. However, there is a tendency for the older basalts from the eastern end of the Walvis Ridge (80-110 m.y. old), the Rio Grande Rise ( 80 m.y. old), Site 525 (70 m.y. old), and possibly for those from Volcanic Units 1, 2, and 3 of Site 527 to have $\mathrm{Zr} / \mathrm{Nb}$ ratios close to 10 . Basalts from the central section, Sites 527 and 528 , have $\mathrm{Zr} / \mathrm{Nb}$ ratios closer to 6 . There are some basalts from the Guyot sction that range from 10 to about 5 .

If the Walvis Ridge originated at a spreading center and represents the trace of a hot spot as the plates separated, then the changing $\mathrm{Zr} / \mathrm{Nb}$ ratio suggests a change in the mantle source with time. The small-scale variation at the different sites and provinces along the Walvis 
G. THOMPSON, S. E. HUMPHRIS

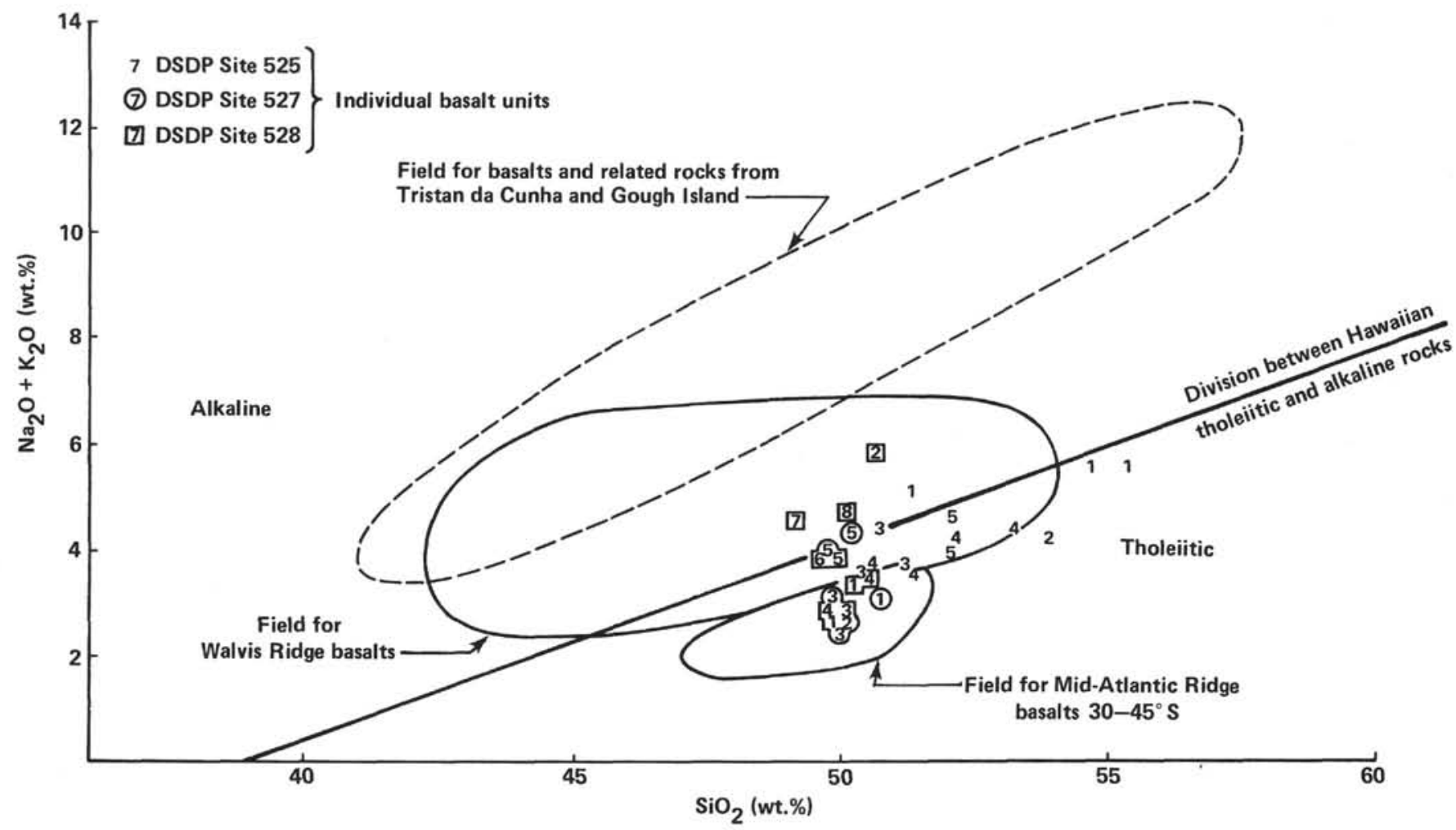

Figure 2. Plot of $\mathrm{Na}_{2} \mathrm{O}+\mathrm{K}_{2} \mathrm{O}$ versus $\mathrm{SiO}_{2}$ for DSDP Leg 74 samples and the fields for related rocks from that region. The numbers in the symbols represent the various volcanic units.

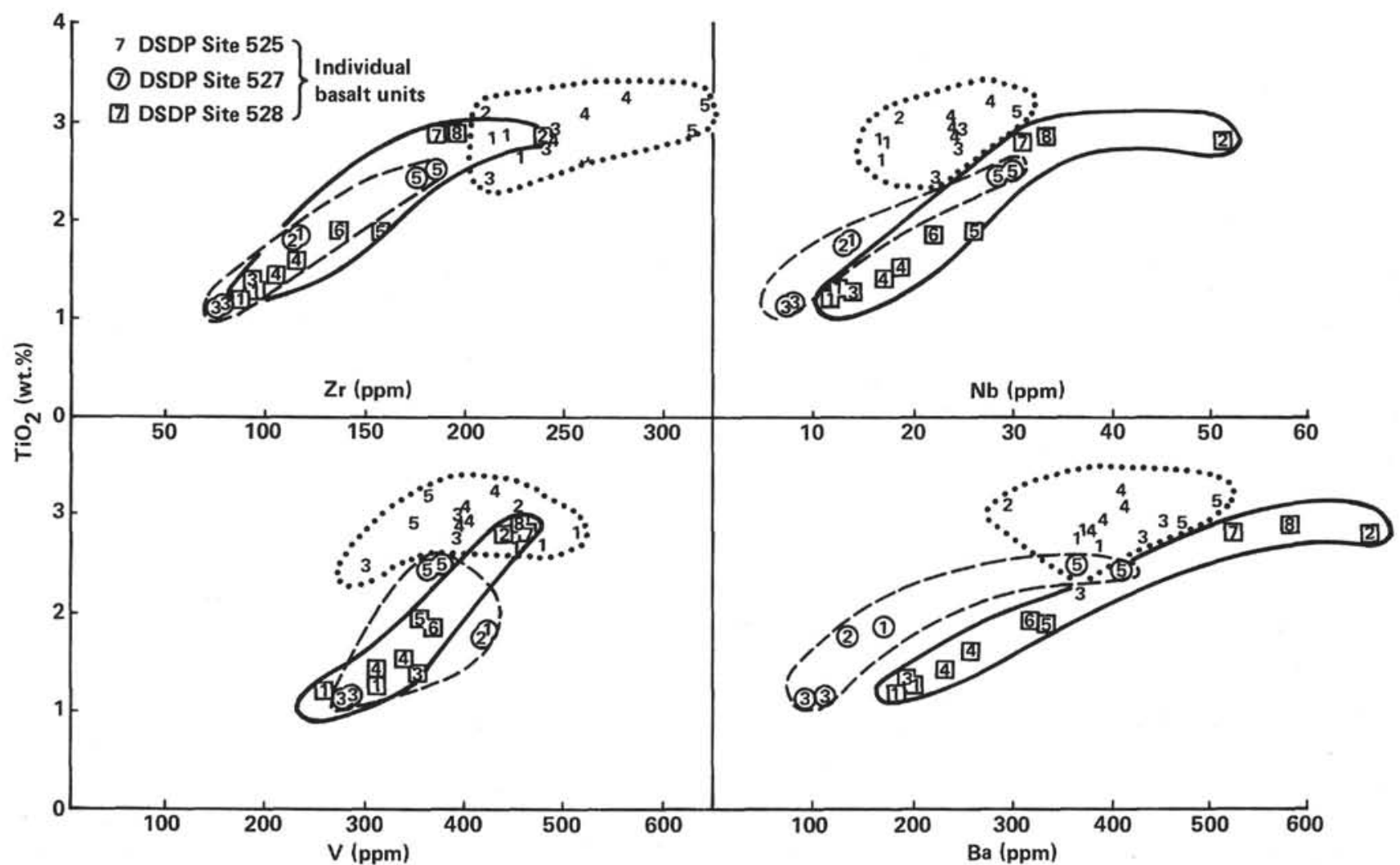

Figure 3. Plots of $\mathrm{TiO}_{2}$ versus $\mathrm{Zr}, \mathrm{Nb}, \mathrm{V}$, and $\mathrm{Ba}$ for Sites 525, 527, and 528. The numbers represent the individual volcanic units. The dotted line field is for Site 525, the dashed line field is for Site 527 and the full line field is for Site 528 . 


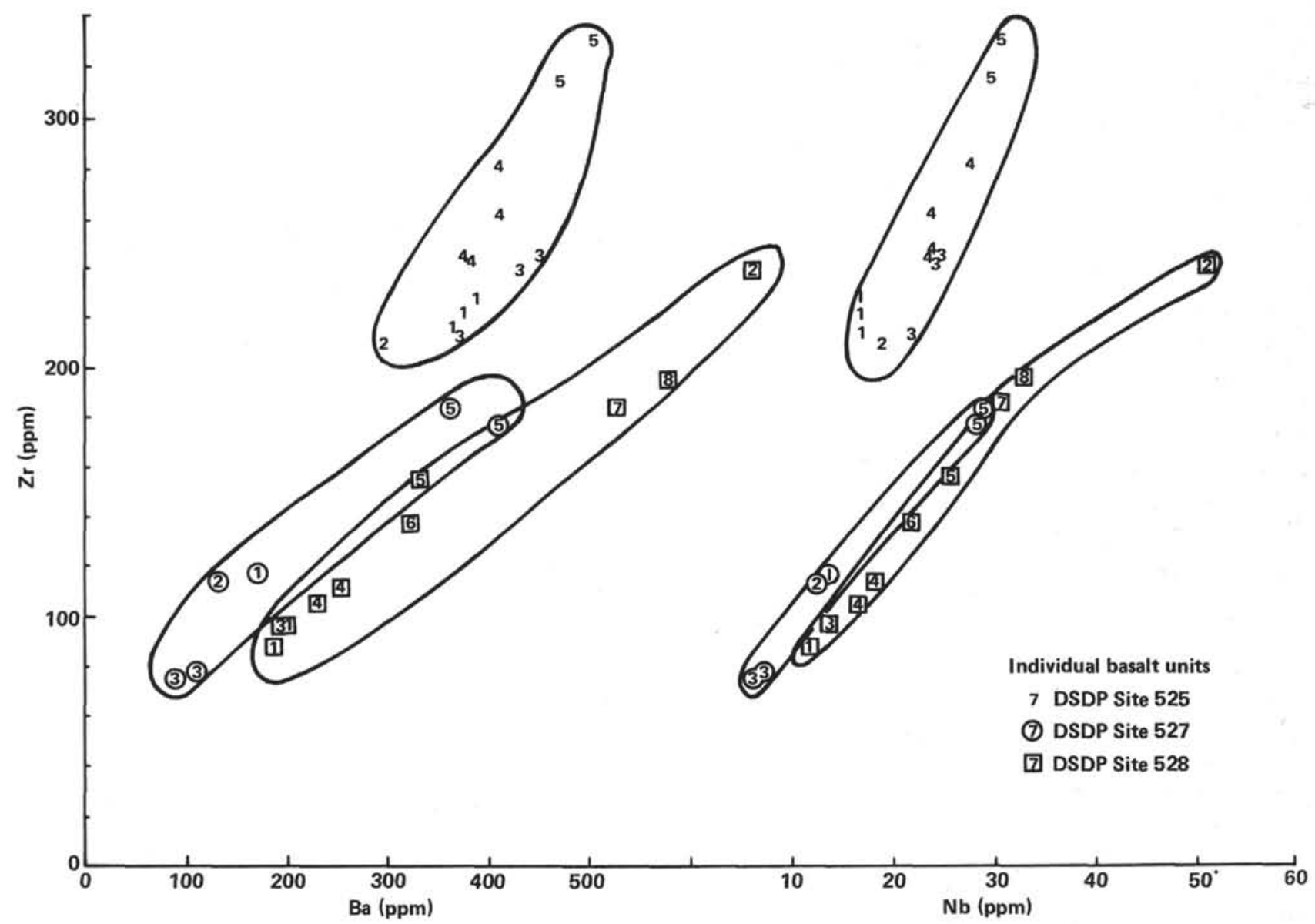

Figure 4. Plots of $\mathrm{Zr}$ versus $\mathrm{Ba}$ and $\mathrm{Nb}$ for Sites 525,527 , and 528 .

Ridge suggests a tendency toward progressively decreasing $\mathrm{Zr} / \mathrm{Nb}$ with time; however, if $\mathrm{Zr} / \mathrm{Nb}$ accurately reflects changes in mantle sources of basalts, clearly some relatively small-scale heterogeneity in the mantle from which the basalts are derived is implied by the compositional variations at individual sites.

Except for those at the latitude of Tristan da Cunha, the basalts at the present-day spreading center at $30^{\circ}$ to $40^{\circ} \mathrm{S}$ are typical MORB. Tristan da Cunha lies $400 \mathrm{~km}$ from the spreading center, but basalts at that latitude (about $37^{\circ} \mathrm{S}$ ) recovered from the spreading center are slightly enriched in incompatible elements compared to typical MORBs (Schilling et at., 1981). They are not as enriched as the Walvis Ridge or Rio Grande Rise basalts and have $\mathrm{Zr} / \mathrm{Nb}$ ratios about 14 to 16 . These data suggest that the present-day hot spot, even though some distance from the ridge axis, does influence the geochemical characteristics of the eruptives at the spreading center. Although Tristan da Cunha and mid-ocean ridge basalts appear to represent two extreme mantle sources, it is not easy to derive the compositions of the Walvis Ridge or Rio Grande Rise basalts by simple mixing of these two possible end-member sources. Although it could produce varying $\mathrm{Zr} / \mathrm{Nb}$ ratios, the extreme enrichment of the Walvis Ridge basalts in other incompatible elements such as Ba, Ti, Sr, and REE (see Fig. 6) im- ply very little if any input of the depleted MORB mantle source.

Thus we conclude that the Walvis Ridge was probably derived from mantle sources of variable composition. These sources have changed dramatically, and possibly progressively, over the past $110 \mathrm{~m} . y$. , although on a shorter time scale they also show evidence of small-scale mantle heterogeneity. Isotopic data on the Walvis Ridge samples will be necessary to test this hypothesis further.

\section{ACKNOWLEDGMENTS}

We thank DSDP for access to Leg 74 core samples and Drs. Mottl and Karson for reviews of the manuscript. Grateful acknowledgment is made to Margaret Sulanowska, Brian Schroeder, and Don Bankston for their help in core sampling and analyses. The figures were drafted by Ms. Sulanowska. This work was supported by NSF grant OCE 80-24629 and is contribution no. 5112 from the Woods Hole Oceanographic Institution.

\section{REFERENCES}

Baker, P. E., Gass, I. G., Harris, P. G., and le Maitre, R. W., 1964. The volcanological report of the Royal Society expedition to Tristan da Cunha, 1962. Phil. Trans. Roy. Soc. London A, 256: 439-575.

Connary, S. D., 1972. Investigations of the Walvis Ridge and environs [Ph.D. dissert.] Columbia University.

Detrick, R. S., Sclater, J. G., and Thiede, J., 1977. The subsidence of aseismic ridges. Earth Planet. Sci. Lett., 34:185-196. 
Detrick, R. S., and Watts, A. B., 1979. An analysis of isostasy in the world's oceans: Part 3-aseismic ridges. J. Geophys. Res., 84: $3637-3653$.

Erlank, A. J., and Kable, E. J. D., 1976. The significance of incompatible elements in Mid-Atlantic Ridge basalts from $45^{\circ} \mathrm{N}$ with particular reference to $\mathrm{Zr} / \mathrm{Nb}$. Contrib. Mineral. Petrol., 54: 281-291.

Fodor, R. V., Husler, J. W., and Kumar, N., 1977. Petrology of volcanic rocks from an aseismic rise: implications for the origin of the Rio Grande Rise, South Atlantic Ocean. Earth Planet. Sci. Lett., 35:225-233.

Fodor, R. V., Keil, K., Husler, J. W., and McKee, E. H., 1977. Petrology and $\mathrm{K}-\mathrm{Ar}$ age of volcanic tuff and ash from the Walvis Seamount Province. DSDP Site 359, Leg 39. In Supko, P. R., Perch-Nielsen, K., et al., Init. Repts. DSDP, 39: Washington, D.C. (U.S. Govt. Printing Office), 525-536.

Frey, F. A., Bryan, W. B., and Thompson, G., 1974. Atlantic Ocean floor: geochemistry and petrology of basalts from Legs 2 and 3 of the Deep Sea Drilling Project. J. Geophys. Res., 79:5507-5527.

Hekinian, R., 1972. Volcanics from the Walvis Ridge in the Southeast Atlantic Ocean. Nature, 239:91-93.

Hekinian, R., and Thompson, G., 1976. Comparative geochemistry of volcanics from rift valleys, transform faults, and aseismic ridges. Contrib. Mineral. Petrol., 57:145-162.

Humphris, S. E., and Thompson, G., 1982. A geochemical study of rocks from the Walvis Ridge, South Atlantic. Chem. Geol., 36: 253-274.
Jen, L. A., 1973. The determination of iron (II) in silicate rocks and minerals. Anal. Chim. Acta, 66:315-318.

Kempe, D. R. C., and Schilling, J. G., 1974. Discovery Tablemount basalt: petrology and geochemistry. Contrib. Mineral. Petrol., 44: 101-115.

Morgan, J., 1971. Convection plumes in the lower mantle. Nature, 230:42-43.

1972. Plate motions and deep mantle convection. Geol. Soc. Am. Mem., 132:7-22.

Schilling, J. G., Kingsley, R., Humphris, S. E., and Thompson, G., 1981. Tristan da Cunha hot spot. Eos (Trans. Am. Geophys. Union) 62:424.

Schroeder, B., Thompson, G., Sulanowska, M., and Ludden, J. N., 1980. Analysis of geologic materials using an automated $x$-ray fluorescence system. X-Ray Spectrom., 9:198-205.

Skinner, N. G., Brown, F. W., and Flanagan, F. J., 1981. The $\mathrm{H}_{2} \mathrm{O}^{+}$ contents of some geochemical standards predicted by a calibration line. Geostandards Newsl., 5:3-12.

Thompson, G., Humphris, S. E., and Schilling, J. G., in press. Petrology and geochemistry of basaltic rocks from Rio Grande Rise, South Atlantic: Deep Sea Drilling Project Leg 72, Hole 516F. In Barker, P. F., Carlson, R. L., Johnson, D. A., et al., Init. Repts. $D S D P, 72$ : Washington, D. C. (U.S. Govt. Printing Office).

Wilson, J. T., 1965. Submarine fracture zones, aseismic ridges and the ICSU line: proposed western margin of the East Pacific Rise. $\mathrm{Na}$ ture, 207:907-911.

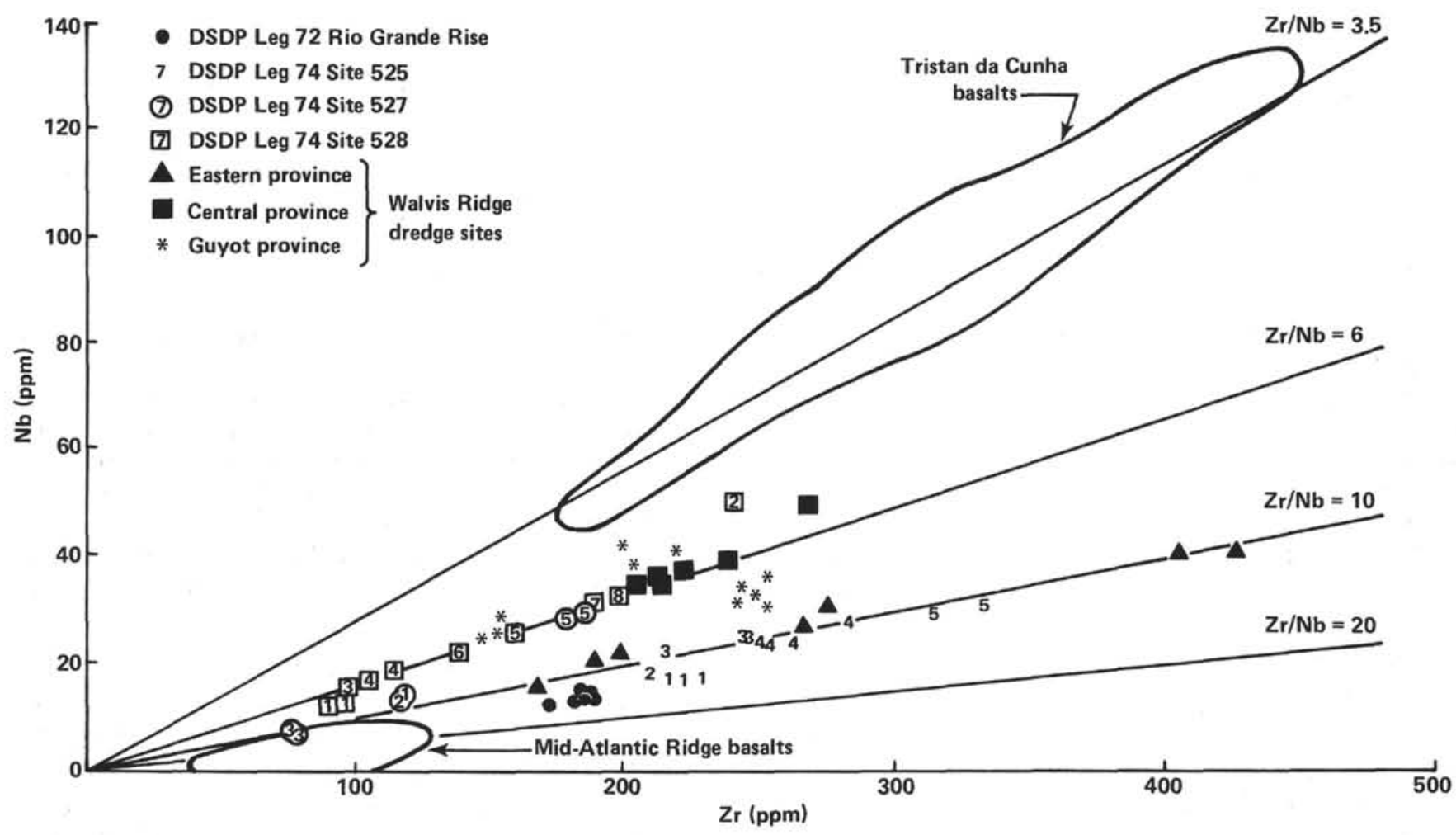

Figure 5. Plot of $\mathrm{Nb}$ versus $\mathrm{Zr}$ for DSDP Leg 74 basalts and for other related rocks. Leg 72 data from Thompson et al. (in press); Walvis Ridge data from Humphris and Thompson (in press). 
Table 8. Comparative analyses of basalts from DSDP Leg 74 Holes 525, 527, and 528 and other basalts from the South Atlantic.

\begin{tabular}{|c|c|c|c|c|c|c|c|c|c|}
\hline & $\begin{array}{c}\text { Hole } 516 \mathrm{~F}^{\mathrm{a}} \\
\text { Rio Grande Rise }\end{array}$ & $\begin{array}{l}\text { Dredge } \mathrm{RC16}{ }^{\mathrm{b}} \\
\text { Rio Grande Rise }\end{array}$ & $\begin{array}{l}\text { Discovery }{ }^{\mathrm{c}} \\
\text { Tablemount }\end{array}$ & $\begin{array}{l}\text { E. Walvis }{ }^{d} \\
\text { Ridge }\end{array}$ & $\begin{array}{c}\text { Tristan da } \\
\text { Cunha }\end{array}$ & $\begin{array}{c}\text { Mid-Atlantic }{ }^{\mathrm{f}} \\
\text { Ridge, S. Atlantic }\end{array}$ & Hole $525^{\mathrm{g}}$ & Hole $527^{\mathrm{h}}$ & Hole $528^{i}$ \\
\hline $\mathrm{SiO}_{2}$ & 50.48 & 47.33 & 51.53 & 51.14 & 46.7 & 50.74 & 52.17 & 50.10 & 49.99 \\
\hline $\mathrm{TiO}_{2}$ & 2.51 & 3.25 & 2.75 & 3.03 & 3.6 & 1.03 & 2.92 & 1.83 & 1.93 \\
\hline $\mathrm{Al}_{2} \mathrm{O}_{3}$ & 15.12 & 14.90 & 15.86 & 16.53 & 17.3 & 15.83 & 17.92 & 15.33 & 16.46 \\
\hline $\mathrm{FeO}^{\mathrm{j}}$ & 12.76 & 9.60 & 9.82 & 11.96 & 10.4 & 9.05 & 9.11 & 12.06 & 11.02 \\
\hline $\mathrm{MnO}$ & 0.17 & 0.17 & 0.16 & & & 0.15 & 0.08 & 0.19 & 0.20 \\
\hline $\mathrm{MgO}$ & 5.23 & 7.19 & 4.99 & 2.37 & 4.7 & 8.78 & 3.49 & 6.40 & 6.26 \\
\hline $\mathrm{CaO}$ & 10.61 & 10.15 & 9.28 & 7.08 & 9.7 & 12.20 & 8.64 & 10.38 & 9.98 \\
\hline $\mathrm{Na}_{2} \mathrm{O}$ & 2.61 & 3.57 & 3.10 & 2.91 & 4.1 & 2.32 & 3.42 & 2.67 & 2.82 \\
\hline $\mathrm{K}_{2} \mathrm{O}$ & 0.28 & 1.73 & 1.43 & 2.19 & 3.0 & 0.06 & 0.94 & 0.59 & 1.01 \\
\hline $\mathrm{P}_{2} \mathrm{O}_{5}$ & 0.21 & 0.75 & 0.43 & & & & 0.30 & 0.25 & 0.28 \\
\hline $\mathrm{R} \bar{b}$ & 6.5 & 2.8 & 22 & & 173 & & 15 & 11 & 16 \\
\hline Sc & 38 & & 22.5 & & & 42 & & & \\
\hline V & 380 & 271 & 400 & 437 & 230 & 233 & 353 & 360 & 366 \\
\hline $\mathrm{Cr}$ & 32 & 146 & 50 & 73 & 28 & 500 & 139 & 45 & 35 \\
\hline Co & 49 & 45 & 40 & 62 & 18 & 49 & 62 & 42 & 43 \\
\hline $\mathrm{Ni}$ & 52 & 106 & 100 & 55 & 10 & 174 & 53 & 45 & 42 \\
\hline $\mathrm{Cu}$ & 198 & 55 & 225 & 110 & & 102 & 79 & 142 & 113 \\
\hline $\mathrm{Zn}$ & 112 & 103 & 227 & & & & 139 & 80 & 79 \\
\hline $\mathrm{Sr}$ & 360 & 928 & $>1000$ & 318 & 1167 & 124 & 482 & 159 & 302 \\
\hline $\mathrm{Ba}$ & 176 & 1156 & 600 & 384 & 913 & 13 & 403 & 197 & 350 \\
\hline$Y$ & 38 & 25 & 50 & 46 & 45 & 35 & 37 & 39 & 35 \\
\hline $\mathrm{Zr}$ & 180 & 293 & 250 & 200 & 325 & 91 & 251 & 125 & 142 \\
\hline $\mathrm{Nb}$ & 13.7 & 70 & & 20 & 112 & $<5$ & 23 & 17 & 24 \\
\hline $\mathrm{La}$ & 14.7 & & 29.4 & 25.1 & 196 & 2.2 & & & \\
\hline $\mathrm{Ce}$ & 36.2 & & & 65 & & 7.1 & & & \\
\hline $\mathrm{Nd}$ & 20.0 & & & 35.2 & & 7.2 & & & \\
\hline Sm & 6.5 & & 7.8 & 8.3 & & 2.41 & & & \\
\hline $\mathrm{Eu}$ & 2.2 & & 2.4 & 2.5 & & 0.93 & & & \\
\hline Gd & 8.5 & & & & & & & & \\
\hline $\mathrm{Tb}$ & 1.2 & & 1.0 & 1.2 & & 0.69 & & & \\
\hline Dy & 7.2 & & & & & & & & \\
\hline $\mathrm{Tm}$ & 0.57 & & & & & & & & \\
\hline $\mathrm{Yb}$ & 3.1 & & 1.5 & 3.3 & & 2.8 & & & \\
\hline $\mathrm{Lu}$ & 0.40 & & 0.29 & 0.53 & & 0.49 & & & \\
\hline
\end{tabular}

a Average of 13 basalts (Thompson et al., in press).

b Mean of 3 basalts, D11A, D12C, and D12A (Fodor, Husler, et al., 1977).

c Fresh basalt (Kempe and Schilling, 1974).

d Mean of 7 basalts (Hekinian, 1972; Hekinian and Thompson, 1976; REE from Frey (unpublished data).

e Average of 10 analyses (Baker et al., 1964).

f Average of 7 basalt glass analyses, DSDP Leg 3 (Frey et al., 1974).

$\mathrm{g}$ Average of 13 basalts, this paper, Tables 2 and 3.

$\mathrm{h}$ Average of 6 basalts, this paper, Tables 4 and 5 .

Average of 10 basalts, this paper, Tables 6 and 7 .

j Total $\mathrm{Fe}$ as $\mathrm{FeO}$. 


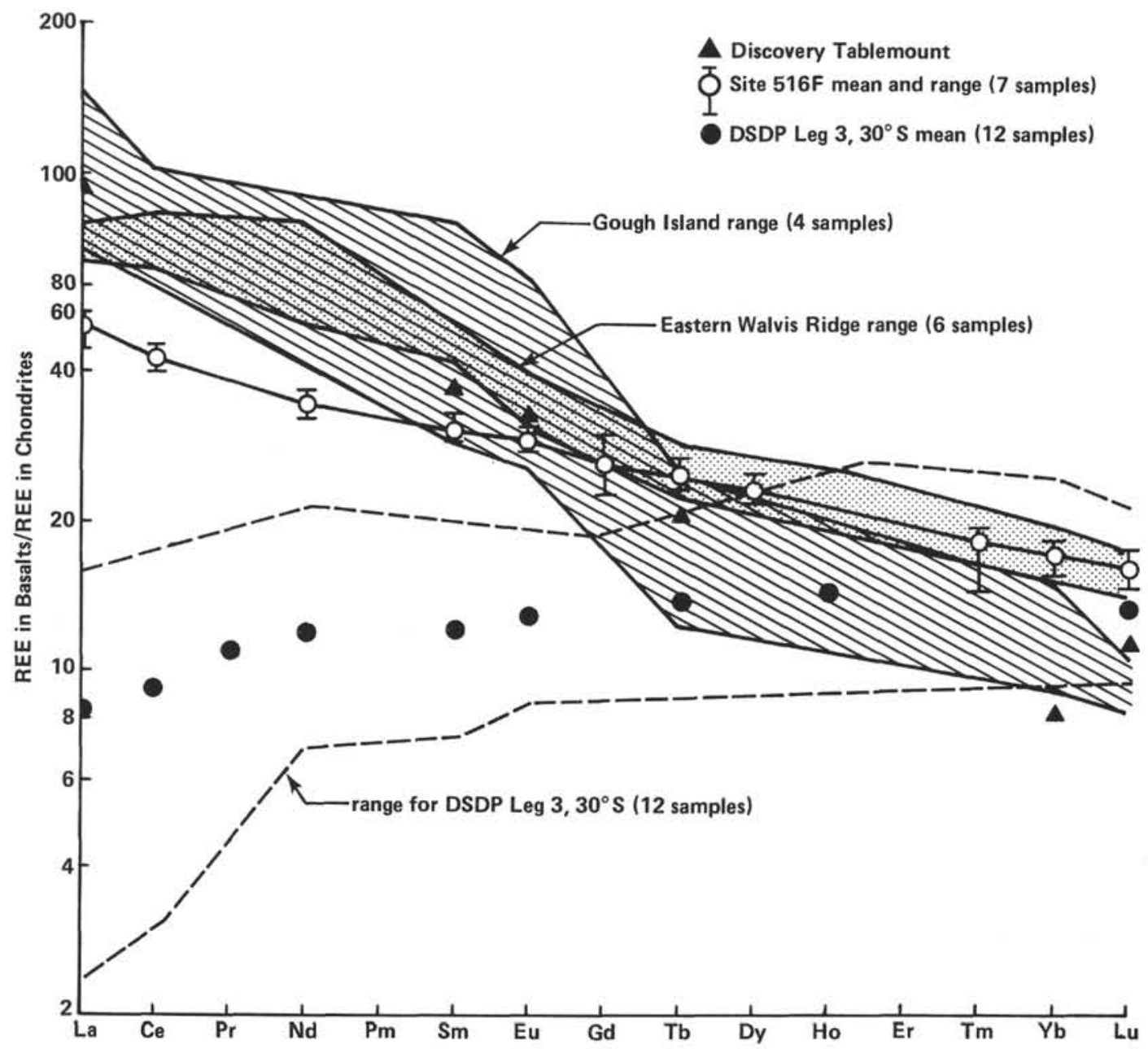

Figure 6. Chondrite-normalized REE data for rocks from the Walvis Ridge and surrounding areas. Figure and data are from Thompson et al. (in press). 